\title{
Toward understanding transcriptional regulatory networks in abiotic stress responses and tolerance in rice
}

\author{
Daisuke Todaka ${ }^{1}$, Kazuo Nakashima ${ }^{1}$, Kazuo Shinozaki ${ }^{2}$ and Kazuko Yamaguchi-Shinozaki ${ }^{1,3^{*}}$
}

\begin{abstract}
Abiotic stress causes loss of crop production. Under abiotic stress conditions, expression of many genes is induced, and their products have important roles in stress responses and tolerance. Progress has been made in understanding the biological roles of regulons in abiotic stress responses in rice. A number of transcription factors (TFs) regulate stress-responsive gene expression. OsDREB1s and OsDREB2s were identified as abiotic-stress responsive TFs that belong to the AP2/ERF family. Similar to Arabidopsis, these DREB regulons were most likely not involved in the abscisic acid (ABA) pathway. OsAREBs such as OsAREB1 were identified as key components in ABAdependent transcriptional networks in rice. OsNAC/SNACs including OsNAC6 were characterized as factors that regulate expression of genes important for abiotic stress responses in rice. Here, we review on the rice abioticstress responses mediated by transcriptional networks, with the main focus on TFs that function in abiotic stress responses and confer stress tolerance in rice.
\end{abstract}

\section{Inroduction}

In most crops, actual yields are only $20 \%$ of attainable yields (Boyer 1982,). In crop production, a dominant factor limiting yield is abiotic stress such as excess or deficient water, high or low temperature, and high salinity (Boyer 1982,). Therefore, improvement of abiotic stress tolerance might increase actual yields extensively in the most crops. As plants are sessile by nature they have evolved adaptive mechanisms against abiotic stress conditions. Recent progress in molecular biology has opened the door to uncovering the adaptive mechanisms at the molecular level in plants (Yamaguchi-Shinozaki \& Shinozaki 2005,, 2006).

A large number of abiotic stress responsive genes have been reported in a variety of plants including rice and Arabidopsis. These genes induced during stress conditions function not only in the protection of cells from stress by production of important metabolic proteins, but also in the regulation of genes, including transcription factors (TFs), for signal transduction in the stress responses. These TFs regulate expression of multiple

\footnotetext{
* Correspondence: kazukoys@jircas.affrc.go.jp

'Biological Resources and Post-harvest Division, Japan International Research Center for Agricultural Sciences, Tsukuba, Ibaraki 305-8686, Japan Full list of author information is available at the end of the article
}

downstream target genes under stress conditions. These regulatory systems are achieved through specific cis-elements in the promoter regions of target genes, which are termed 'regulons'. Several regulons involved in abiotic stress responses have been identified in Arabidopsis (Nakashima et al. 2009,; Qin et al. 2011,). The dehydration-responsive element binding protein 1 (DREB1)/Crepeat binding factor $(\mathrm{CBF})$ regulon functions in the cold stress response, whereas the DREB2 regulon acts in heat and osmotic stress responses (Mizoi et al. 2011,). The abscisic acid (ABA) responsive element (ABRE) binding protein (AREB)/ABRE binding factor (ABF) regulon functions in ABA-dependent gene expression under osmotic stress conditions (Fujita et al. 2011,). In addition, the NAC regulon is shown to be involved in osmotic stress responses (Nakashima et al. 2009).

Rice (Oryza sativa L.) is a staple crop for more than half of the world's population. In addition to this social importance, rice shows promise as an excellent model plant for studies on cereals. The rice genome is onesixth the size of the maize genome and 40 times smaller than the wheat genome (IRGSP 2005,). The small genome size has enriched a set of resources available for molecular biological studies (Jung et al. 2008). Rice serves also as a model plant for studies of biofuel 
perennial grasses, such as switchgrass and Miscanthus (Kellogg 2001,). Transcriptomic analyses of rice treated with abiotic stresses have been carried out (Cooper et al. 2003,; Hazen et al. 2005,; Lan et al. 2005,; Rabbani et al. 2003,; Ray et al. 2011; Wang et al. 2007; Zhou et al. 2007). These analyses clarified a large number of stressresponsive genes, which can be divided into two groups. One group is a signaling component that regulates gene expression in the stress responses, including protein kinases and TFs. The other group is a functional component that directly protects plant cells against the stress, including enzymes in metabolic pathways, aquaporins, and late embryogenesis abundant (LEA) proteins. Efforts to identify and characterize these stress-responsive genes have uncovered several abiotic stress regulatory networks in rice. Here, we review the rice abiotic stress responses mediated by transcriptional networks, and focus on TFs that function in abiotic stress responses and confer stress tolerance in rice.

\section{Overview of abiotic stress responses in rice}

Numerous stress-responsive genes have been identified in transcriptomic analyses of rice treated with abiotic stresses Rabbani et al. (2003),. identified 36, 62, and 57 genes that were induced by cold, drought, and high salinity, respectively. These included genes encoding calmodulin, catalase, LEA protein, metallothionein-like protein, transcription factors such as zinc finger, NAC, and Myb, lipoxygenase, and sugar transporter protein Wang et al. (2007),. compared comprehensive expression profiles between genotypes of upland rice, which is resistant to water stress, and lowland rice, which is susceptible to water stress, that were subjected to PEG treatment. The authors found 13\% of genes expressed in leaves and 7\% of genes expressed in roots showed differential expression between the two genotypes. Genes highly expressed in upland rice included TFs, proteins conferring detoxification or protection against oxidative stress, and proteins that maintain cell turgor. Genes highly expressed in lowland rice were involved in the degradation of cellular components. Zhou et al. (2007), examined expression patterns under drought or high salinity stress in the shoot, flag leaf, and panicle. The majority of genes expressed in response to the stress treatments were organ-specific. For example, the number of genes expressed specifically in the drought-treated shoot, flag leaf, and panicle were 1020, 301, and 448, respectively, whereas the number of genes expressed in all of these organs was 42. The latter genes included protein kinases, chlorophyll a/b binding protein, LEA proteins, and transcription factors such as zinc finger type and homeobox type. Thus, expression of thousands of genes is regulated by a variety of transcriptional cascades when rice plants are subjected to abiotic stresses.
Transcriptomic analysis of other cereal crops, including barley (Oztur et al. 2002,; Talame et al. 2007,), maize (Andjelkovic \& Thompson 2006,; Luo et al. 2010;; Yu \& Setter 2003,; Zheng et al. 2004,) and sorghum (Buchanan et al. 2005), has shown that thousands of genes are upor down-regulated under abiotic stress conditions. Therefore, analysis of transcriptional regulatory systems is important to clarify abiotic stress responses and tolerance. Some regulons, such as DREB, AREB, and NAC, have been analyzed extensively in rice (Figure 1) and Arabidopsis. Evidence is emerging that similar, but not identical, regulons exist in rice compared to Arabidopsis. Regulons modulated by DREB1, DREB2, AREB, NAC, and other TFs, and other stress-responsive genes in rice are reviewed in the following sections. TFs described in this paper are listed in Table 1.

\section{DREB1/CBF regulon}

Arabidopsis DREB1-type genes DREB1A, DREB1B and $D R E B 1 C$ were identified as transcription factors that bind to a cis-acting element, DRE/CRT. The DRE/CRT contains the core sequence A/GCCGAC important for regulation of gene expression in response to drought, high salinity, and cold stresses in Arabidopsis (Yamaguchi-Shinozaki \& Shinozaki 2005). The expression of $D R E B 1 A, D R E B 1 B$ and DREB1C was induced by cold stress and the three encoded DREB1 proteins were major transcriptional activators required for cold-inducible gene expression. Transgenic Arabidopsis overexpressing DREB1-type genes driven by the Cauliflower mosaic virus (CaMV) $35 \mathrm{~S}$ promoter displayed strong tolerance to drought, high salinity, and freezing with growth retardation (Yamaguchi-Shinozaki \& Shinozaki 2006 ,). Use of a stress-inducible promoter minimized the growth retardation. More than 100 downstream

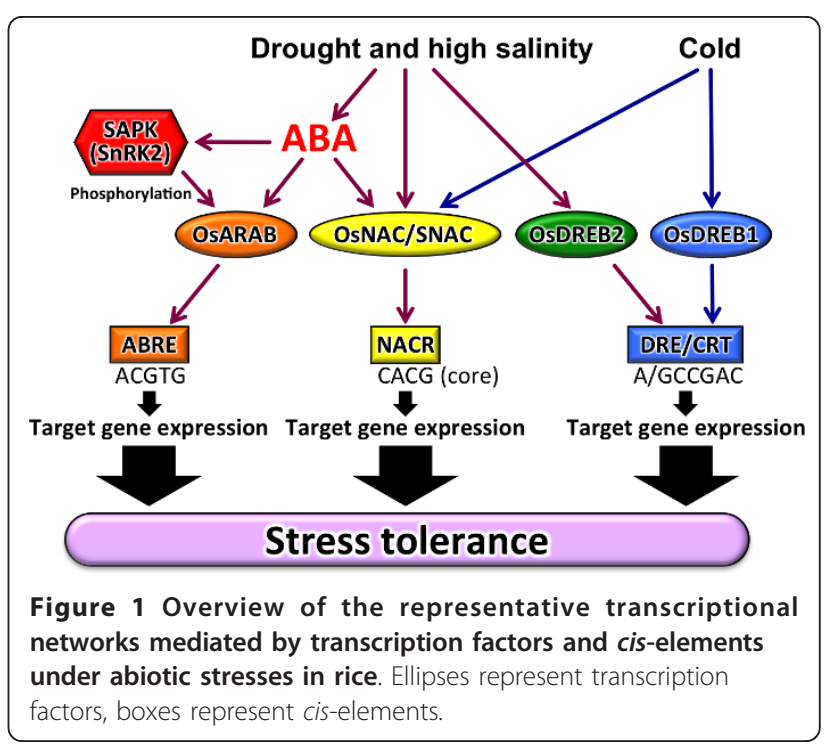


Table $1 \mathrm{~A}$ list of transcription factors (TFs) described in this paper

\begin{tabular}{|c|c|c|c|}
\hline Gene name & Tolerance in OX (mutant) & Phenomena or roles in stress response & Reference \\
\hline \multicolumn{4}{|l|}{ OsDREB1 TFs } \\
\hline OsDREB1A & Drought $\uparrow$, Salt $\uparrow$, Cold $\uparrow$ & $\begin{array}{l}\text { Accumulation of proline and regulation of stress-responsive gene } \\
\text { expression }\end{array}$ & (Ito et al. 2006) \\
\hline OsDREB1B & Drought $\uparrow$, Salt $\uparrow$, Cold $\uparrow$ & Induction of the TF expression in cold stress & (Ito et al. 2006) \\
\hline OsDREB1F & Drought $\uparrow$, Salt $\uparrow$, Cold $\uparrow$ & Regulation of ABA-responsive gene expression & (Wang et al. 2008) \\
\hline OsDREB1G & Drought $\uparrow$ & Binding to the DRE element & (Chen et al. 2008) \\
\hline \multicolumn{4}{|l|}{ OsDREB2 TFs } \\
\hline OsDREB2A & Not reported & Induction of the TF expression in heat, drought and salt stresses & (Matsukura et al. 2010) \\
\hline OsDREB2B & Drought $\uparrow$, Heat $\uparrow$ & Regulation of gene expression via stress-induced alternative splicing & (Matsukura et al. 2010) \\
\hline \multicolumn{4}{|l|}{ OSAREB TFS } \\
\hline TRAB1 & Not reported & Regulation of gene expression via phosphorylation in response to $A B A$ & $\begin{array}{l}\text { (Hobo et al. 1999, Kagaya et al. } \\
\text { 2002) }\end{array}$ \\
\hline OsABF2 & Drought $\downarrow$, Salt $\downarrow$, Cold $\downarrow$ & Modulation of gene expression through an ABA-dependent pathway & (Hossain et al. 2010) \\
\hline OsABI5 & Salt $\downarrow$, (Salt $\uparrow)$ & Regulation of adaptive stress response and plant fertility & (Zou et al. 2008) \\
\hline OsbZIP23 & Drought $\uparrow$, Salt $\uparrow$ & $\begin{array}{l}\text { Regulation of expression of genes involved in stress response and } \\
\text { tolerance }\end{array}$ & (Xiang et al. 2008) \\
\hline ABL1 & Not reported & Modulation of $\mathrm{ABA}$ and auxin responses & (Yang et al. 2011) \\
\hline OsbZIP72 & Drought $\uparrow$ & Regulation of $A B A$ responsive gene expression & (Lu et al. 2009) \\
\hline \multicolumn{4}{|l|}{ OsNAC TFs } \\
\hline $\begin{array}{l}\text { OsNAC6/ } \\
\text { SNAC2 }\end{array}$ & Drought $\uparrow$, Salt $\uparrow$ & Regulation of abiotic and biotic stress responsive gene expression & $\begin{array}{l}\text { (Nakashima et al. 2007, } \mathrm{Hu} \\
\text { et al. 2008) }\end{array}$ \\
\hline OsNAC5 & Salt $\uparrow$ & Regulation of OsLEA3 expression & $\begin{array}{l}\text { (Takasaki et al. 2010,; Song et al. } \\
\text { 2011) }\end{array}$ \\
\hline SNAC1 & Drought $\uparrow$ & $\begin{array}{l}\text { Increase of stomatal closure and expression of genes involved in } \\
\text { stress tolerance }\end{array}$ & (Hu et al. 2006) \\
\hline OsNAC10 & Drought $\uparrow$ & Enhancement of grain yield under drought conditions & (Jeong et al. 2010) \\
\hline \multicolumn{4}{|l|}{ Other TFs } \\
\hline AP37 & Drought $\uparrow$ & Enhancement of grain yield & (Oh et al. 2009) \\
\hline AP59 & Drought $\uparrow$ & Spikelet disruption & (Oh et al. 2009) \\
\hline ZFP252 & Drought $\uparrow$, Salt $\uparrow$ & Accumulation of proline and sugars & (Xu et al. 2008) \\
\hline ZFP245 & Drought $\uparrow$, Cold $\uparrow$ & Elevation of ROS enzyme activity & (Huang et al. 2009a) \\
\hline ZFP179 & Salt $\uparrow$ & Accumulation of proline and sugars & (Sun et al. 2010) \\
\hline DST & (Drought $\uparrow$, Salt) & Regulation H202-induced stomatal closure & (Huang et al. 2009b) \\
\hline OsWRKY45-2 & Salt $\downarrow$ (Salt $\uparrow)$ & Regulation of $A B A$ sensitivity & (Tao et al. 2009) \\
\hline OsTIFY11a & Salt $\uparrow$, Mannitol $\uparrow$ & Regulation seed germination & (Ye et al. 2009) \\
\hline Osmyb4 & Chilling $\uparrow$ & The TF decreases membrane injury & $\begin{array}{l}\text { (Vannini et al. 2004; Park et al. } \\
\text { 2010) }\end{array}$ \\
\hline OsMYB3R-2 & Drought $\uparrow$, Salt $\uparrow$, Cold $\uparrow$ & Regulation of $\mathrm{G} 2 / \mathrm{M}$ phase specific gene expression & (Dai et al. 2007) \\
\hline
\end{tabular}

target genes were identified and the most of these genes possessed the DRE core motif in their promoter regions (Yamaguchi-Shinozaki \& Shinozaki 2006).

The rice genome contains at least ten DREB1-type genes, among which OsDREB1A and OsDREB1B are induced by cold stress (Dubouzet et al. 2003). Overexpression of OSDREB1A induced strong expression of stressresponsive genes in transgenic Arabidopsis plants, which resulted in not only improved stress tolerance to high-salinity and freezing stresses, but also growth retardation of plants under non-stress growth conditions (Dubouzet et al. 2003). This result indicates OsDREB1A shows functional similarity to DREB1A. In addition, transgenic rice overexpressing OsDREB1A and OsDREB1B also showed growth retardation under non-stress conditions and improved tolerance to drought, high-salinity and cold stress (Ito et al. 2006). The transgenic rice plants accumulated osmoprotectants, including proline and a variety of sugars, under control conditions as occurred in the transgenic Arabidopsis (Ito et al. 2006). Target stress-inducible genes of OsDREB1A were identified by microarray analysis and encode proteins thought to function in stress tolerance in the plants, which is consistent with the target genes of DREB1 proteins in Arabidopsis. These observations indicate the DREB1 regulon is conserved in rice and DREB1-type genes are useful for improvement of tolerance to environmental stresses in transgenic plants including rice. 
OsDREB1F is induced by salt, drought, and cold stresses, and also ABA application (Wang et al. 2008). Transgenic rice plants harboring the OsDREB1F gene showed enhanced stress tolerance to salt, drought, and cold stress. Overexpression of OsDREB1F in Arabidopsis enhanced expression of stress-inducible genes, such as $r d 29 B$ and $R A B 18$, that contain the DRE/CRT element in their promoter regions, which suggests that OsDREB1F might also participate in expression of osmotic stress responsive genes through the ABAdependent signaling pathway. Overexpression of OsDREB1G also elevates drought tolerance (Chen et al. 2008). These results indicate the DREB1 regulon in rice is one of the master regulatory system in abiotic stress responses.

\section{DREB2 regulon}

The Arabidopsis DREB2 protein also has a conserved ERF/AP2 DNA-binding domain and recognizes the DRE sequence. However, the expression pattern and transcriptional regulatory mechanism of DREB2 differed from those of DREB1. DREB2A and DREB2B were induced by drought, high salinity and high temperature. The encoded DREB2 proteins function as major transcriptional activators required for expression of genes inducible by these stresses. Overexpression of DREB2A in transgenic Arabidopsis neither caused growth retardation nor improved stress tolerance (Yamaguchi-Shinozaki \& Shinozaki 2006). A negative regulatory domain was indicated to exist in the central region of DREB2A and deletion of this region transforms DREB2A into a constitutively active form (DREB2A CA) (Qin et al. 2011,; Mizoi et al. 2011). Overexpression of DREB2A $C A$ in transgenic Arabidopsis increased expression of many stress-inducible genes and resulted in enhanced tolerance to drought, salt and heat stresses (Mizoi et al. 2011,). The protein stability mediated by ubiquitin E3 ligases is important for activation of DREB2A (Qin et al. 2011).

Rice has at least four $D R E B 2$ homologues, among which $O s D R E B 2 A$ and $O s D R E B 2 B$ are induced by drought, high salinity and heat stresses (Matsukura et al. 2010). OsDREB2B is also induced by cold stress. OsDREB2B expression is regulated by alternative splicing that generates two-types of transcripts, namely functional and nonfunctional forms. The functional transcript encodes the full-length protein, whereas the nonfunctional form has a stop codon within the fulllength protein. Although the nonfunctional form of transcripts accumulates under non-stress conditions, the functional form of transcripts is induced by abiotic stresses. The alternative splicing of DREB2-type genes was observed also in other monocotyledonous plants such as barley (Xue \& Loveridge 2004,), wheat (Egawa et al. 2006,) and maize (Qin et al. 2007). Transgenic Arabidopsis plants overexpressing the functional form of OsDREB2B displayed enhanced expression of DREB2A target genes and improved tolerance to drought and heat stresses (Matsukura et al. 2010). These analyses indicate $O s D R E B 2 B$ is a key gene that encodes the DREB2-type TF that functions in stress-responsive gene expression in rice. Transgenic Arabidopsis plants overexpressing the functional form of maize $Z m D R E B 2 A$ showed improved tolerance to drought and heat stresses (Qin et al. 2007). Microarray analysis showed that the up-regulated genes in Arabidopsis plants overexpressing $Z m D R E B 2 A$ were similar to those in Arabidopsis plants overexpressing DREB2A CA (Qin et al. 2007,; Sakuma et al. 2006). Collectively, the function of DREB2-type genes in different plants is similar but the regulatory systems of DREB2-type gene expression are most likely different between monocotyledons and dicotyledons; the former requires RNA processing, whereas the latter changes protein stability.

\section{AREB regulon}

Abscisic Acid acts as a crucial signal molecule in abiotic stress responses (Fujita et al. 2011). The ABA content is increased by abiotic stresses, and leads to expression of numerous genes. Application of exogenous ABA also stimulates a myriad of genes. ABRE was identified as a cis-acting element conserved in promoter regions of ABA-inducible genes. Arabidopsis cDNAs that encode bZIP-type TFs were screened as ABRE-binding proteins (Yamaguchi-Shinozaki \& Shinozaki 2006). Among these genes, $A R E B 1 / A B F 2, A R E B 2 / A B F 4$, and $A B F 3$ were reported to be induced by $\mathrm{ABA}$ and osmotic stress in vegetative tissues (Fujita et al. 2011,). Evidence indicates that activation of AREB1 needs ABA-dependent posttranscriptional modification. The ABA-activated SnRK2 protein kinases phosphorylate the AREB1 protein (Furihata et al. 2006). Transgenic Arabidopsis plants overexpressing the phosphorylated active form of AREB1 showed enhanced expression of a number of ABA-inducible genes (Furihata et al. 2006). The ABA-activated phosphorylation of AREB/ABFs was completely impaired in the SnRK2 triple mutant, srk2d srk2e srk2i (Fujii et al. 2009,; Fujii \& Zhu 2009). The down-regulated genes in the srk2d srk2e srk2 $i$ and areb1 areb2 abf3 triple mutants largely overlapped in ABA-dependent expression, which supports the view that SRK2D/ $\mathrm{E} / \mathrm{I}$ regulate $\mathrm{AREBs}$ in $\mathrm{ABA}$ signaling in response to osmotic stress. (Fujita et al. 2009).

The rice TRANSCRIPTION FACTOR RESPONSIBLE FOR ABA REGULATION1 (TRAB1) shows high homology to Arabidopsis AREB2/ABF4. Expression of TRAB1 was up-regulated by ABA treatment (Hobo et al. 1999,). TRAB1 is phosphorylated rapidly in response to ABA 
treatment (Kagaya et al. 2002). One of the rice bZIPtype TFs, OsABF2, was shown to be induced by drought, high salinity, cold and oxidative stresses, and ABA treatment (Hossain et al. 2010). A T-DNA insertion mutant of OsABF2 showed increased sensitivity to abiotic stresses compared to wild-type plants. The loss of function approach suggests that OsABF2 positively functions in abiotic stress signaling. Another rice bZIP-type TF, OsABI5, was isolated from rice panicles (Zou et al. 2008). Expression of OsABI5 was induced by ABA and high salinity, but it was down-regulated by drought and cold stresses in rice seedlings. Transgenic rice plants overexpressing OsABI5 showed high sensitivity to salt stress. In contrast, antisense OsABI5 transgenic rice plants showed increased stress tolerance but decreased fertility (Zou et al. 2008,). These results indicate OsABI5 might regulate adaptation to stress and plant fertility. (Nijhawan et al. 2008) surveyed the rice genome for bZIP family proteins and analyzed the expression of 89 OsbZIP genes. Their microarray analysis indicated 26 genes were up-regulated and 11 genes were down-regulated under dehydration, salinity, and/or cold conditions.

The function of several abiotic stress-inducible rice bZIP-type TFs has been studied in transgenic rice plants and identification of their downstream genes has been attempted by microarray analyses. Expression of $O s b$ ZIP23 was induced by drought, high salinity and ABA (Xiang et al. 2008). Transgenic rice overexpressing OsbZIP23 exhibited improved tolerance to drought and high-salinity stresses. In contrast, a null mutant for this gene showed decreased tolerance to high-salinity and drought stress. Microarray analyses revealed 37 possible OsbZIP23-specific target genes, which showed changes of reverse expression level in the overexpressor and mutant. ABI5-Like1 (ABL1) was expressed in a number of tissues and its expression was induced by $A B A$ and indole-3-acetic acid treatments, and by drought and salinity stresses (Yang et al. 2011). The abl1 mutant showed suppressed ABA responses. Microarray analysis showed that a large proportion of down-regulated genes in the abl1 mutant are involved in stress responses. ABL1 also regulated a series of ABRE-containing WRKY family genes. In addition, the abl1 mutant showed hypersensitivity to exogenous indole-3-acetic acid, and altered expression of genes related to auxin metabolism or signaling. These mutant analyses suggest that ABL1 modulates ABA and auxin responses. Transgenic rice plants overexpressing OsbZIP72 showed hypersensitivity to $A B A$, elevated levels of expression of ABA-responsive genes such as $L E A$ s, and enhanced drought tolerance ( $\mathrm{Lu}$ et al. 2009). These observations suggest rice AREB homologues regulate ABA-inducible gene expression, similar to the functions of Arabidopsis AREB proteins.
Ten SnRK2 protein kinases have been characterized in rice (Kobayashi et al. 2004,). All family members are activated by hyperosmotic stress. Three genes of the family were also activated by ABA and these ABA-activated SnRK2 proteins can phosphorylate TRAB1 (Kobayashi et al. 2005,). OSRK1 was isolated and encodes a $41.8-\mathrm{kD}$ protein kinase of the SnRK2 family (Chae et al. 2007). In vitro kinase assays demonstrated that OSRK1 can phosphorylate both itself and generic substrates. OREB1, a rice ABRE-binding factor, was phosphorylated in vitro by OSRK1 at multiple sites of different functional domains. Ectopic expression of OSRK1 in transgenic tobacco caused reduced sensitivity to $A B A$ in seed germination and root elongation. These findings indicate OSRK1 is associated with ABA signaling, possibly through the phosphorylation of the $A B F$ family in vivo. Collectively, these findings suggest the ABA-activated SnRK2 protein kinases phosphorylate and activate the AREB/ABF-type proteins in rice, which leads to the enhanced expression of a number of genes involved in abiotic stress responses and tolerance.

\section{NAC regulon}

NAC-type transcription factors that regulate expression of abiotic stress responsive genes were isolated initially from Arabidopsis. In Arabidopsis, three NACs (ANAC019, ANAC055, and ANAC072) were isolated as TFs that regulate expression of a salt- and droughtinduced gene, ERD1 (Yamaguchi-Shinozaki \& Shinozaki 2006). Expression of the three NAC genes was enhanced under salt and drought stress conditions. Microarray analysis of transgenic Arabidopsis plants overexpressing these NAC genes showed several stress-inducible genes were up-regulated in the transgenic plants. These upregulated genes caused significantly increased tolerance to drought in the transgenic lines (Yamaguchi-Shinozaki \& Shinozaki 2006).

In rice several $N A C$ genes are reported to be induced by drought, high salinity, and cold stresses. OsNAC6/ $S N A C 2$ encodes a NAC transcription factor in rice $(\mathrm{Hu}$ et al. 2008,; Nakashima et al. 2007). Expression of OsNAC6/SNAC2 is induced by cold, drought and high salinity and also is enhanced by jasmonic acid, wounding and blast disease. Transgenic rice plants overexpressing OsNAC6 showed enhanced tolerance to dehydration and high salinity stresses, although the plants showed growth retardation and low yield under the non-stress condition. These negative effects were minimized in transgenic rice plants driven by a stressinducible promoter. Microarray analyses using the OsNAC6 overexpressors clarified the downstream genes, which included many stress-inducible genes. Among these genes, two genes, one of which was a gene that encoded peroxidase, were activated in a transient 
transactivation assay. These results indicate OsNAC6 functions as a transcriptional activator in both abiotic and biotic stress responses. Furthermore, a rice gene that encodes a histone deacetylase, OsHDAC1, epigenetically represses OsNAC6 expression (Chung et al. 2009).

OsNAC5 expression was induced by dehydration, cold, ABA, and MeJA treatments (Takasaki et al. 2010). The growth of rice plants overexpressing OsNAC5 was similar to that of control plants under non-stress conditions. The OsNAC5 overexpressors showed improved tolerance to high salinity. Microarray analyses using the OsNAC5 overexpressors identified downstream genes, which included OsLEA3. OsNAC5 was shown to bind the NAC recognition core sequence (CACG) in the OsLEA3 promoter region in a gel mobility shift assay. Song et al. (2011) reported that RNA interference (RNAi) transgenic rice plants with reduced OsNAC5 expression showed less tolerance to abiotic stresses than control plants, whereas overexpression of OsNAC5 enhanced abiotic stress tolerance. It was also shown that accumulation of proline and soluble sugars was positively correlated with OsNAC5 expression level. SNAC1 was identified as a key component that functions in stress responses in rice (Hu et al. 2006). SNAC1 expression was induced by drought, salt, cold and ABA treatment. Overexpression of SNAC1 improved drought tolerance. The SNAC1 overexpressors did not show any negative effects on growth and productivity. Crystal structure analysis of the SNAC1 protein revealed the overall architecture and two loop regions essential for DNA binding (Chen et al. 2011). OsNAC10 was expressed in roots and panicles under non-stress conditions (Jeong et al. 2010). The expression was induced by drought, high salinity, and ABA. Root-specific overexpression of OsNAC10 enhanced tolerance and grain yield in the field under drought conditions.

These results indicate that, in addition to the DREB and AREB regulons, the NAC regulon is also important for transcriptional networks of abiotic stress responses both in monocotyledons and dicotyledons.

\section{Other TF regulons}

Other TFs are reported to act in abiotic stress responses in rice. These include AP2/ERFs (AP37, AP59), $\mathrm{C}_{2} \mathrm{H}_{2}$ zinc fingers (ZFP252, ZFP245, ZFP179 and DST), a WRKY (OsWRKY45), a TIFY (OsTIFY11a), and MYBs (Osmyb4, and OsMYB3R-2). Transgenic rice plants overexpressing AP37 or AP59 showed increased tolerance to drought (Oh et al. 2009). Overexpression of AP37 improved grain yield under drought conditions, but overexpression of AP59 caused spikelet disruption and resulted to lower yield than WT. Overexpression of ZFP252, a $\mathrm{C}_{2} \mathrm{H}_{2}$-type zinc finger protein, increased salt and drought stress tolerance, and elevated expression of stress-responsive genes such as OsDREB1A (Xu et al. 2008). Transgenic rice plants overexpressing ZFP245 showed significant tolerance to cold and drought stresses (Huang et al. 2009a). In the overexpressors, the activities of reactive oxygen species-scavenging enzymes were elevated under stress conditions. Overexpression of ZFP179 increased the levels of proline and soluble sugars, leading to enhanced salt tolerance (Sun et al. 2010). DST mutants displayed enhanced tolerance to drought and salt stresses, which was mediated by $\mathrm{H}_{2} \mathrm{O}_{2}$ induced stomatal closure (Huang et al. 2009b). Os WRKY45-1 and OsWRKY45-2 were isolated from japonica and indica rice, respectively (Tao et al. 2009,). Expression of both genes was induced by cold stress, but was reduced under drought stress (Tao et al. 2011). Transgenic rice plants overexpressing OsWRKY45-1 showed reduced ABA sensitivity, whereas overexpressors of OsWRKY45-2 displayed increased ABA sensitivity and salt stress tolerance. Expression of the OsTIFY11a gene was induced by cold, drought and salt stresses (Ye et al. 2009). Overexpressors of OsTIFY11a showed improved salt and mannitol tolerance. Overexpression of Osmyb4, a cold-inducible MYB TF gene, enhanced cold and freezing tolerance in transgenic Arabidopsis plants (Vannini et al. 2004). Transgenic rice plants overexpressing Osmyb4 showed enhanced chilling tolerance (Park et al. 2010). The plants exhibited decreased membrane injury and a high germination rate under a low temperature. Transcriptome analysis using Osmyb4 overexpressors showed that expression of genes associated with cellular defense and rescue, metabolism and development were changed in the overexpressors. Challenges ectopically expressing Osmyb4 were performed in other crops such as apple (Pasquali et al. 2008) and Osteospermum ecklonis (Laura et al. 2010,), an ornamental and perennial plant native to South Africa. These plants showed enhanced tolerance to cold and drought stresses through phenylpropanoid metabolism and biosynthesis of compatible osmolytes. OsMYB3R-2 was also reported to be a stress-inducible MYB TF (Dai et al. 2007). Transgenic Arabidopsis plants overexpressing OsMYB3R-2 showed elevated tolerance to cold, drought and high-salinity stresses with high expression levels of stress-inducible genes such as DREB2A and COR15a. These findings indicate that a variety of TFs function in abiotic stress responses and tolerance in rice. These TFs could be useful for development of transgenic crops with enhanced tolerance to abiotic stresses.

\section{Abiotic stress tolerance mechanisms of transgenic rice plants or mutants with altered TF expression}

One of the mechanisms that contribute to abiotic stress tolerance is the accumulation of osmoprotectants in plant cells. Osmoprotectant compounds include amino acids, 
onium compounds and polyoles/sugars. These compounds are produced in cells subjected to abiotic stresses and stabilize proteins and cell membranes. Transgenic Arabidopsis plants overexpressing DREB1A showed up-regulated expression of genes that encode osmoprotectant biosynthesis proteins, which leads to the accumulation of osmoprotectants, such as myo-inositol, sucrose, galactinol and raffinose (Maruyama et al. 2009). This finding indicates that DREB1A functions as a regulator of osmoprotectant biosynthesis to enhance stress tolerance. Transgenic rice plants overexpressing DREB1A also accumulated Pro and sugars. Although a metabolic change in OsDREB1A overexpressors has not been identified successfully, it is suggested that DREB1A orthologues can function in the osmoprotectant biosynthesis pathways in rice similar to those in Arabidopsis.

LEA proteins are implicated in the mechanism of desiccation tolerance (Goyal et al. 2005). Overexpression of OsLEA3-1 in rice improved grain yield under drought conditions (Xiao et al. 2007). Some genes that encode LEA proteins, including OsLEA3-1, were up-regulated in OsbZIP23 overexpressors (Xiang et al. 2008). One mechanism that contributes to the enhanced tolerance in OsbZIP23 overexpressors may be the result of upregulated $L E A$ gene expression. OsLEA3-1 was also upregulated in OsNAC5 overexpressors (Takasaki et al. 2010). These authors demonstrated that OsNAC5 and OsNAC6 can bind to the promoter region of OsLEA3-1.

The relationship among each TF in abiotic stress responses is not fully understood. We compared OsDREB1A, AP59, OsbZIP23, SNAC1, OsNAC5 and OsNAC6 downstream genes identified by microarray analyses. No downstream genes were up-regulated in all overexpressors of these TFs. Most downstream genes were specifically up-regulated in each overexpressor. For example, 72 downstream genes in AP59 overexpressors and 156 downstream genes in OsNAC6 overexpressors did not overlap, and only two genes, Os10g0419400 and Os01g0795200, which encode acireductone dioxygenase and subtilisin-like serine proteinase, respectively, overlapped. Only one gene, Os10g0450900, which encodes glycine-rich protein, was up-regulated in three overexpressors, namely OsDREB1A, OsbZIP23 and OsNAC6 overexpressors. These results suggest that OsDREB1A, AP59, OsbZIP23, SNAC1, OsNAC5 and OsNAC6 TFs function independently in abiotic stress responses with a specific set of downstream genes.

\section{Conclusions}

Significant progress has been made in understanding the biological roles of several regulons in abiotic stress responses in rice. TFs play important roles in the signaling cascades. OsDREB1A, OsDREB1B, OsDREB1F, OsDREB2A, and OsDREB2B were identified as abiotic stress responsive DREB-type TFs in rice. Similar to Arabidopsis, these regulons were most likely not involved in the ABA signaling pathway. Several TFs were characterized that regulate expression of ABA-responsive genes in rice. OsABF2, OsbZIP23, ABL1, OsbZIP72, TRAB1, and OsABI5 are thought to function as key components in ABA-dependent transcriptional networks in rice. The NAC-type TFs OsNAC6, SNAC1, OsNAC5, and OsNAC10 are also important regulons in abiotic stress responses in rice.

Ray et al. (2011) reported that 1,563 and 1,746 genes were up- or down-regulated, respectively, under waterdeficit conditions, respectively. Among these genes, members of zinc finger TFs such as $\mathrm{C} 2 \mathrm{C} 2, \mathrm{C} 3 \mathrm{H}$, LIM, PHD, ZF-HD, and TFs that encode GeBP, jumonji, MBF1, and ULT were newly identified as TFs differentially expressed under water stress. The role of these TFs in the stress response remains unclear.

Development of genetically modulated rice plants that show enhanced tolerance and yield under adverse conditions is still a challenge. One key objective is to identify the genes responsible for abiotic stress tolerance in rice. Variations exist in tolerance mechanisms among plant species. Understanding the crosstalk between different stress signal transduction pathways is also important. Because plants are highly adaptable and have many mechanisms to survive environmental stresses, dissection of abiotic stress responses will be an important clue to enhance crop yield.

\section{Acknowledgements}

This work was supported by grants from the Ministry of Agriculture, Forestry and Fisheries of Japan (in part by Genomics for Agricultural Innovation, Development of Abiotic Stress Tolerant Crops by DREB Genes) and the Program for Promotion of Basic and Applied Researches for Innovations in Bio-oriented Industry (BRAIN) of Japan.

\section{Author details}

${ }^{1}$ Biological Resources and Post-harvest Division, Japan International Research Center for Agricultural Sciences, Tsukuba, Ibaraki 305-8686, Japan ${ }^{2}$ RIKEN Plant Science Center, Yokohama, Kanagawa 230-0045, Japan ${ }^{3}$ Laboratory of Plant Molecular Physiology, Graduate School of Agricultural and Life Sciences, The University of Tokyo, Bunkyo-ku, Tokyo 113-8657, Japan

Authors' contributions

DT wrote the paper, KN drew a figure, and KS and KY-S designed research. All authors read and approved the final manuscript.

\section{Competing interests}

The authors declare that they have no competing interests.

Received: 4 November 2011 Accepted: 8 March 2012

Published: 8 March 2012

\section{References}

Andjelkovic V, Thompson R (2006) Changes in gene expression in maize kernel in response to water and salt stress. Plant Cell Rep 25:71-79. doi:10.1007/ s00299-005-0037-x.

Boyer JS (1982) Plant productivity and environment. Science 218:443-448. doi:10.1126/science.218.4571.443 
Buchanan CD, Lim S, Salzman RA, Kagiampakis I, Morishige DT, Weers BD., et al (2005) Sorghum bicolor's transcriptome response to dehydration, high salinity and ABA. Plant Mol Biol 58:699-720. doi:10.1007/s11103-005-7876-2.

Chae MJ, Lee JS, Nam MH, Cho K, Hong JY, Yi SA., et al (2007) A rice dehydration-inducible SNF1-related protein kinase 2 phosphorylates an abscisic acid responsive element-binding factor and associates with ABA signaling. Plant Mol Biol 63:151-169

Chen JQ, Meng XP, Zhang Y, Xia M, Wang XP (2008) Over-expression of OsDREB genes lead to enhanced drought tolerance in rice. Biotechnol Lett 30:2191-2198. doi:10.1007/s10529-008-9811-5.

Chen Q, Wang Q, Xiong L, Lou Z (2011) A structural view of the conserved domain of rice stress-responsive NAC1. Protein Cell 2:55-63. doi:10.1007/ s13238-011-1010-9.

Chung PJ, Kim YS, Jeong JS, Park SH, Nahm BH, Kim JK (2009) The histone deacetylase OSHDAC1 epigenetically regulates the OsNAC6 gene that controls seedling root growth in rice. Plant J 59:764-776. doi:10.1111/j.1365313X.2009.03908.X.

Cooper B, Clarke JD, Budworth P, Kreps J, Hutchison D, Park S., et al (2003) A network of rice genes associated with stress response and seed development. Proc Natl Acad Sci USA 100:4945-4950. doi:10.1073/ pnas. 0737574100 .

Dai X, Xu Y, Ma Q, Xu W, Wang T, Xue Y., et al (2007) Overexpression of an R1R2R3 MYB gene, OsMYB3R-2, increases tolerance to freezing, drought, and salt stress in transgenic Arabidopsis. Plant Physiol 143:1739-1751. doi:10.1104/pp.106.094532.

Dubouzet JG, Sakuma Y, Ito Y, Kasuga M, Dubouzet EG, Miura S., et al (2003) OsDREB genes in rice, Oryza sativa L., encode transcription activators that function in drought-, high-salt- and cold-responsive gene expression. Plant J 33:751-763. doi:10.1046/j.1365-313X.2003.01661.x.

Egawa C, Kobayashi F, Ishibashi M, Nakamura T, Nakamura C, Takumi S (2006) Differential regulation of transcript accumulation and alternative splicing of a DREB2 homolog under abiotic stress conditions in common wheat. Genes Genet Syst 81:77-91. doi:10.1266/ggs.81.77.

Fujii H, Zhu JK (2009) Arabidopsis mutant deficient in 3 abscisic acid-activated protein kinases reveals critical roles in growth, reproduction, and stress. Proc Natl Acad Sci USA 106:8380-8385. doi:10.1073/pnas.0903144106.

Fujii H, Chinnusamy V, Rodrigues A, Rubio S, Antoni R, Park SY., et al (2009) In vitro reconstitution of an abscisic acid signalling pathway. Nature 462:660-664. doi:10.1038/nature08599.

Fujita Y, Fujita M, Shinozaki K, Yamaguchi-Shinozaki K (2011) ABA-mediated transcriptional regulation in response to osmotic stress in plants. J Plant Res 124:509-525. doi:10.1007/s10265-011-0412-3.

Fujita Y, Nakashima K, Yoshida T, Katagiri T, Kidokoro S, Kanamori N., et al (2009) Three SnRK2 protein kinases are the main positive regulators of abscisic acid signaling in response to water stress in Arabidopsis. Plant Cell Physiol 50:2123-2132. doi:10.1093/pcp/pcp147.

Furihata T, Maruyama K, Fujita Y, Umezawa T, Yoshida R, Shinozaki K., et al (2006) Abscisic acid-dependent multisite phosphorylation regulates the activity of a transcription activator AREB1. Proc Natl Acad Sci USA 103:1988-1993. doi:10.1073/pnas.0505667103.

Goyal K, Walton LJ, Tunnacliffe A (2005) LEA proteins prevent protein aggregation due to water stress. Biochem J 388:151-157. doi:10.1042/ BJ20041931.

Hazen SP, Pathan MS, Sanchez A, Baxter I, Dunn M, Estes B., et al (2005) Expression profiling of rice segregating for drought tolerance QTLs using a rice genome array. Funct Integr Genomics 5:104-116. doi:10.1007/s10142004-0126-X.

Hobo T, Kowyama Y, Hattori T (1999) A bZIP factor, TRAB1, interacts with VP1 and mediates abscisic acid-induced transcription. Proc Natl Acad Sci USA 96:15348-15353. doi:10.1073/pnas.96.26.15348.

Hossain MA, Cho Jl, Han M, Ahn CH, Jeon JS, An G., et al (2010) The ABREbinding bZIP transcription factor OSABF2 is a positive regulator of abiotic stress and ABA signaling in rice. J Plant Physiol 167:1512-1520. doi:10.1016/j. jplph.2010.05.008.

Hu H, Dai M, Yao J, Xiao B, Li X, Zhang Q., et al (2006) Overexpressing a NAM, ATAF, and CUC (NAC) transcription factor enhances drought resistance and salt tolerance in rice. Proc Natl Acad Sci USA 103:12987-12992. doi:10.1073/ pnas.0604882103.

Hu H, You J, Fang Y, Zhu X, Qi Z, Xiong L (2008) Characterization of transcription factor gene SNAC2 conferring cold and salt tolerance in rice. Plant Mol Biol 67:169-181. doi:10.1007/s11103-008-9309-5.
Huang J, Sun SJ, Xu DQ, Yang X, Bao YM, Wang ZF., et al (2009) Increased tolerance of rice to cold, drought and oxidative stresses mediated by the overexpression of a gene that encodes the zinc finger protein ZFP245. Biochem Biophys Res Commun 389:556-561. doi:10.1016/j.bbrc.2009.09.032.

Huang XY, Chao DY, Gao JP, Zhu MZ, Shi M, Lin HX (2009) A previously unknown zinc finger protein, DST, regulates drought and salt tolerance in rice via stomatal aperture control. Genes Dev 23:1805-1817. doi:10.1101/ gad.1812409.

IRGSP (2005) The map-based sequence of the rice genome. Nature 436:793-800. doi:10.1038/nature03895.

Ito Y, Katsura K, Maruyama K, Taji T, Kobayashi M, Seki M., et al (2006) Functional analysis of rice DREB1/CBF-type transcription factors involved in coldresponsive gene expression in transgenic rice. Plant Cell Physiol 47:141-153

Jeong JS, Kim YS, Baek KH, Jung H, Ha SH, Do Choi Y., et al (2010) Root-specific expression of OsNAC10 improves drought tolerance and grain yield in rice under field drought conditions. Plant Physiol 153:185-197. doi:10.1104/ pp.110.154773.

Jung KH, An G, Ronald PC (2008) Towards a better bowl of rice: assigning function to tens of thousands of rice genes. Nat Rev Genet 9:91-101

Kagaya Y, Hobo T, Murata M, Ban A, Hattori T (2002) Abscisic acid-induced transcription is mediated by phosphorylation of an abscisic acid response element binding factor, TRAB1. Plant Cell 14:3177-3189. doi:10.1105/ tpc.005272.

Kellogg EA (2001) Evolutionary history of the grasses. Plant Physiol 125:1198-1205. doi:10.1104/pp.125.3.1198.

Kobayashi Y, Yamamoto S, Minami H, Kagaya Y, Hattori T (2004) Differential activation of the rice sucrose nonfermenting1-related protein kinase2 family by hyperosmotic stress and abscisic acid. Plant Cell 16:1163-1177. doi:10.1105/tpc.019943.

Kobayashi Y, Murata M, Minami H, Yamamoto S, Kagaya Y, Hobo T., et al (2005) Abscisic acid-activated SNRK2 protein kinases function in the gene-regulation pathway of ABA signal transduction by phosphorylating ABA response element-binding factors. Plant J 44:939-949. doi:10.1111/j.1365313X.2005.02583.X.

Lan L, Li M, Lai Y, Xu W, Kong Z, Ying K., et al (2005) Microarray analysis reveals similarities and variations in genetic programs controlling pollination/ fertilization and stress responses in rice (Oryza sativa L.). Plant Mol Biol 59:151-164. doi:10.1007/s11103-005-3958-4.

Laura M, Consonni R, Locatelli F, Fumagalli E, Allavena A, Coraggio I., et al (2010) Metabolic response to cold and freezing of Osteospermum ecklonis overexpressing Osmyb4. Plant Physiol Biochem 48:764-771. doi:10.1016/j. plaphy.2010.06.003.

Lu G, Gao C, Zheng X, Han B (2009) Identification of OsbZIP72 as a positive regulator of ABA response and drought tolerance in rice. Planta 229:605-615. doi:10.1007/s00425-008-0857-3.

Luo M, Liu J, Lee RD, Scully BT, Guo B (2010) Monitoring the expression of maize genes in developing kernels under drought stress using oligo-microarray. J Integr Plant Biol 52:1059-1074. doi:10.1111/j.1744-7909.2010.01000.x.

Maruyama K, Takeda M, Kidokoro S, Yamada K, Sakuma Y, Urano K., et al (2009) Metabolic pathways involved in cold acclimation identified by integrated analysis of metabolites and transcripts regulated by DREB1A and DREB2A. Plant Physiol 150:1972-1980. doi:10.1104/pp.109.135327.

Matsukura S, Mizoi J, Yoshida T, Todaka D, Ito Y, Maruyama K., et al (2010) Comprehensive analysis of rice DREB2-type genes that encode transcription factors involved in the expression of abiotic stress-responsive genes. Mol Genet Genomics 283:185-196. doi:10.1007/s00438-009-0506-y.

Mizoi J, Shinozaki K, Yamaguchi-Shinozaki K (2011) AP2/ERF family transcription factors in plant abiotic stress responses. Biochim Biophys Acta 1819:86-96

Nakashima K, Tran LS, Van Nguyen D, Fujita M, Maruyama K, Todaka D., et al (2007) Functional analysis of a NAC-type transcription factor OsNAC6 involved in abiotic and biotic stress-responsive gene expression in rice. Plant J 51:617-630. doi:10.1111/j.1365-313X.2007.03168.X.

Nakashima K, Ito Y, Yamaguchi-Shinozaki K (2009) Transcriptional regulatory networks in response to abiotic stresses in Arabidopsis and grasses. Plant Physiol 149:88-95. doi:10.1104/pp.108.129791.

Nijhawan A, Jain M, Tyagi AK, Khurana JP (2008) Genomic survey and gene expression analysis of the basic leucine zipper transcription factor family in rice. Plant Physiol 146:333-350

Oh SJ, Kim YS, Kwon CW, Park HK, Jeong JS, Kim JK (2009) Overexpression of the transcription factor AP37 in rice improves grain yield under drought conditions. Plant Physiol 150:1368-1379. doi:10.1104/pp.109.137554. 
Oztur ZN, Talame V, Deyholos M, Michalowski CB, Galbraith DW, Gozukirmizi N., et al (2002) Monitoring large-scale changes in transcript abundance in drought- and salt-stressed barley. Plant Mol Biol 48:551-573. doi:10.1023/ A:1014875215580.

Park MR, Yun KY, Mohanty B, Herath V, Xu F, Wijaya E., et al (2010) Supra-optimal expression of the cold-regulated OsMyb4 transcription factor in transgenic rice changes the complexity of transcriptional network with major effects on stress tolerance and panicle development. Plant Cell Environ 33:2209-2230. doi:10.1111/j.1365-3040.2010.02221.x.

Pasquali G, Biricolti S, Locatelli F, Baldoni E, Mattana M (2008) Osmyb4 expression improves adaptive responses to drought and cold stress in transgenic apples. Plant Cell Rep 27:1677-1686. doi:10.1007/s00299-008-0587-9.

Qin F, Kakimoto M, Sakuma Y, Maruyama K, Osakabe Y, Tran LS., et al (2007) Regulation and functional analysis of ZmDREB2A in response to drought and heat stresses in Zea mays L. Plant J 50:54-69. doi:10.1111/j.1365313X.2007.03034.X.

Qin F, Shinozaki K, Yamaguchi-Shinozaki K (2011) Achievements and challenges in understanding plant abiotic stress responses and tolerance. Plant Cell Physiol 52:1569-1582. doi:10.1093/pcp/pcr106.

Rabbani MA, Maruyama K, Abe H, Khan MA, Katsura K, Ito Y., et al (2003) Monitoring expression profiles of rice genes under cold, drought, and highsalinity stresses and abscisic acid application using cDNA microarray and RNA gel-blot analyses. Plant Physiol 133:1755-1767. doi:10.1104/ pp.103.025742.

Ray S, Dansana PK, Giri J, Deveshwar P, Arora R, Agarwal P., et al (2011) Modulation of transcription factor and metabolic pathway genes in response to water-deficit stress in rice. Funct Integr Genomics 11:157-178. doi:10.1007/ s10142-010-0187-y.

Sakuma Y, Maruyama K, Osakabe Y, Qin F, Seki M, Shinozaki K., et al (2006) Functional analysis of an Arabidopsis transcription factor, DREB2A, involved in drought-responsive gene expression. Plant Cell 18:1292-1309. doi:10.1105/ tpc.105.035881.

Song SY, Chen Y, Chen J, Dai XY, Zhang WH (2011) Physiological mechanisms underlying OsNAC5-dependent tolerance of rice plants to abiotic stress. Planta 234:331-345. doi:10.1007/s00425-011-1403-2.

Sun SJ, Guo SQ, Yang X, Bao YM, Tang HJ, Sun H., et al (2010) Functional analysis of a novel Cys2/His2-type zinc finger protein involved in salt tolerance in rice. J Exp Bot 61:2807-2818. doi:10.1093/jxb/erq120.

Takasaki H, Maruyama K, Kidokoro S, Ito Y, Fujita Y, Shinozaki K, et al (2010) The abiotic stress-responsive NAC-type transcription factor OSNAC5 regulates stress-inducible genes and stress tolerance in rice. Mol Genet Genomics 284:173-183. doi:10.1007/s00438-010-0557-0.

Talame V, Ozturk NZ, Bohnert HJ, Tuberosa R (2007) Barley transcript profiles under dehydration shock and drought stress treatments: a comparative analysis. J Exp Bot 58:229-240

Tao Z, Liu H, Qiu D, Zhou Y, Li X, Xu C., et al (2009) A pair of allelic WRKY genes play opposite roles in rice-bacteria interactions. Plant Physiol 151:936-948. doi:10.1104/pp.109.145623.

Tao Z, Kou Y, Liu H, Li X, Xiao J, Wang S (2011) OsWRKY45 alleles play different roles in abscisic acid signalling and salt stress tolerance but similar roles in drought and cold tolerance in rice. J Exp Bot 62:4863-4874. doi:10.1093/jxb/ err144.

Vannini C, Locatelli F, Bracale M, Magnani E, Marsoni M, Osnato M., et al (2004) Overexpression of the rice Osmyb4 gene increases chilling and freezing tolerance of Arabidopsis thaliana plants. Plant J 37:115-127. doi:10.1046/ j.1365-313X.2003.01938.X.

Wang H, Zhang H, Gao F, Li J, Li Z (2007) Comparison of gene expression between upland and lowland rice cultivars under water stress using CDNA microarray. Theor Appl Genet 115:1109-1126. doi:10.1007/s00122-007-0637-7.

Wang Q, Guan Y, Wu Y, Chen H, Chen F, Chu C (2008) Overexpression of a rice OsDREB1F gene increases salt, drought, and low temperature tolerance in both Arabidopsis and rice. Plant Mol Biol 67:589-602. doi:10.1007/s11103008-9340-6.

Xiang Y, Tang N, Du H, Ye H, Xiong L (2008) Characterization of OsbZIP23 as a key player of the basic leucine zipper transcription factor family for conferring abscisic acid sensitivity and salinity and drought tolerance in rice. Plant Physiol 148:1938-1952. doi:10.1104/pp.108.128199.

Xiao B, Huang Y, Tang N, Xiong L (2007) Over-expression of a LEA gene in rice improves drought resistance under the field conditions. Theor Appl Genet 115:35-46. doi:10.1007/s00122-007-0538-9.
Xu DQ, Huang J, Guo SQ, Yang X, Bao YM, Tang HJ., et al (2008) Overexpression of a TFIIIA-type zinc finger protein gene ZFP252 enhances drought and salt tolerance in rice (Oryza sativa L.). FEBS Lett 582:1037-1043. doi:10.1016/j. febslet.2008.02.052.

Xue GP, Loveridge CW (2004) HvDRF1 is involved in abscisic acid-mediated gene regulation in barley and produces two forms of AP2 transcriptional activators, interacting preferably with a CT-rich element. Plant J 37:326-339

Yamaguchi-Shinozaki K, Shinozaki K (2005) Organization of cis-acting regulatory elements in osmotic- and cold-stress-responsive promoters. Trends Plant Sci 10:88-94. doi:10.1016/j.tplants.2004.12.012.

Yamaguchi-Shinozaki K, Shinozaki K (2006) Transcriptional regulatory networks in cellular responses and tolerance to dehydration and cold stresses. Annu Rev Plant Biol 57:781-803. doi:10.1146/annurev.arplant.57.032905.105444.

Yang X, Yang YN, Xue L, Zou MJ, Liu JY, Chen F., et al (2011) Rice ABI5-Like1 regulates abscisic acid and auxin responses by affecting the expression of ABRE-containing genes. Plant Physiol 156:1397-1409. doi:10.1104/ pp.111.173427.

Ye H, Du H, Tang N, Li X, Xiong L (2009) Identification and expression profiling analysis of TIFY family genes involved in stress and phytohormone responses in rice. Plant Mol Biol 71:291-305. doi:10.1007/s11103-009-9524-8.

Yu LX, Setter TL (2003) Comparative transcriptional profiling of placenta and endosperm in developing maize kernels in response to water deficit. Plant Physiol 131:568-582. doi:10.1104/pp.014365.

Zheng J, Zhao J, Tao Y, Wang J, Liu Y, Fu J., et al (2004) Isolation and analysis of water stress induced genes in maize seedlings by subtractive PCR and CDNA macroarray. Plant Mol Biol 55:807-823

Zhou J, Wang X, Jiao Y, Qin Y, Liu X, He K., et al (2007) Global genome expression analysis of rice in response to drought and high-salinity stresses in shoot, flag leaf, and panicle. Plant Mol Biol 63:591-608. doi:10.1007/ s11103-006-9111-1.

Zou M, Guan Y, Ren H, Zhang F, Chen F (2008) A bZIP transcription factor, OsABI5, is involved in rice fertility and stress tolerance. Plant Mol Biol 66:675-683. doi:10.1007/s11103-008-9298-4.

doi:10.1186/1939-8433-5-6

Cite this article as: Todaka et al:: Toward understanding transcriptional regulatory networks in abiotic stress responses and tolerance in rice. Rice 2012 5:6.

\section{Submit your manuscript to a SpringerOpen ${ }^{\circ}$ journal and benefit from:}

- Convenient online submission

- Rigorous peer review

- Immediate publication on acceptance

- Open access: articles freely available online

- High visibility within the field

- Retaining the copyright to your article

Submit your next manuscript at $\gg$ springeropen.com 